Utah State University

DigitalCommons@USU

$1-1-2006$

\title{
An Update of Sounding of the Atmosphere Using Broadband Emission Radiometry (SABER) Calibration
}

Joseph J. Tansock

James M. Russell III

Martin G. Mlynczak

Larry L. Gordley

Chris Brown

Greg Paxton

See next page for additional authors

Follow this and additional works at: https://digitalcommons.usu.edu/sdl_pubs

\section{Recommended Citation}

Tansock, Joseph J.; Russell, James M. III; Mlynczak, Martin G.; Gordley, Larry L.; Brown, Chris; Paxton, Greg; and McMichaels, Patrick, "An Update of Sounding of the Atmosphere Using Broadband Emission Radiometry (SABER) Calibration" (2006). Space Dynamics Lab Publications. Paper 129.

https://digitalcommons.usu.edu/sdl_pubs/129

This Article is brought to you for free and open access by the Space Dynamics Lab at DigitalCommons@USU. It has been accepted for inclusion in Space Dynamics Lab Publications by an authorized administrator of DigitalCommons@USU. For more information, please contact digitalcommons@usu.edu.

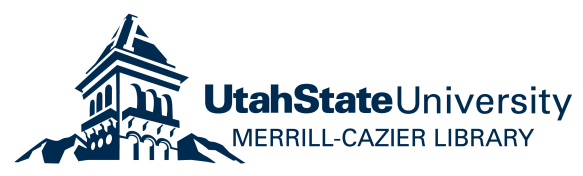


Authors

Joseph J. Tansock, James M. Russell III, Martin G. Mlynczak, Larry L. Gordley, Chris Brown, Greg Paxton, and Patrick McMichaels 


\title{
An Update of Sounding of the Atmosphere using Broadband Emission Radiometry (SABER) Calibration
}

\author{
Joseph J. Tansock ${ }^{\mathrm{a}}$, James M. Russell III ${ }^{\mathrm{b}}$, Martin G. Mlynczak ${ }^{\mathrm{c}}$, Larry L. Gordley ${ }^{\mathrm{d}}$, Chris Brown ${ }^{\mathrm{d}}$, \\ Greg Paxton ${ }^{\mathrm{d}}$, Patrick McMichaels ${ }^{\mathrm{d}}$ \\ ${ }^{a}$ Space Dynamics Laboratory, Utah State University, 1695 N. Research Park Way, N. Logan, UT \\ 84341-1947 \\ ${ }^{\mathrm{b}}$ Center for Atmospheric Sciences, Hampton University, Hampton, VA 23668 \\ 'NASA Langley Research Center, 11 Langley Blvd., Hampton, VA 23681-2199 \\ ${ }^{\mathrm{d} G A T S}$ Incorporated, 11864 Canon Blvd., \#101, Newport News, VA 23606
}

\begin{abstract}
The sounding of the atmosphere using broadband emission radiometry (SABER) instrument is a 10-channel infrared $(1.27-16.9 \mu \mathrm{m})$ radiometer launched on the TIMED (Thermosphere, Ionosphere, Mesosphere Energetics, and Dynamics) satellite in December 2001 from Vandenburg Air Force Base. SABER measures earthlimb emissions and characterizes infrared radiation, allowing calculation of atmospheric temperature and composition (ozone, water vapor, and carbon dioxide), as well as solar and chemical heating rates and infrared cooling rates. Although SABER focuses on the unexplored $60-180 \mathrm{~km}$ region, it makes measurements covering the $10-350 \mathrm{~km}$ altitude region. Ground calibration testing was completed in September 1999. Subsequent data analyses and report generation were completed in June, 2000. This paper provides a brief overview of instrument design, calibration planning, ground calibration testing, and results. Also included is an assessment of nearly five years of post launch validation and calibration maintenance. Using SABER as an example, conclusions are given regarding the benefit of a detailed calibration approach and how it enhances the quality of science data and mission success.
\end{abstract}

Keywords: TIMED, SABER, IR, instrument, calibration, validation

\section{INTRODUCTION}

This paper describes the calibration of the SABER infrared radiometer. SABER is an earthlimb-scanning radiometer and is one of four payload instruments on the TIMED satellite. TIMED was successfully launched December 7, 2001. After nearly five years on-orbit (initial mission goal was two years), SABER continues to make high-quality earthlimb measurements.

Ground calibration testing was completed at Space Dynamics Laboratory/Utah State University Research Foundation (USURF/SDL) in September 1999. Subsequent results are documented in a SABER Calibration Report, ${ }^{1}$ and summarized in publication. ${ }^{2}$

The SABER ground calibration generated a calibration equation and radiometric model, which describes the overall responsivity of the sensor in terms of separate radiometric parameters. ${ }^{3}$ To account for changes in responsivity, the radiance responsivity was monitored during on-orbit operations by viewing in-flight calibration (IFC) sources. The IFC sources were calibrated using SABER as a transfer radiometer to transfer the calibration of ground-based blackbodies to IFC sources.

The SABER ground calibration facility consisted of a ground support equipment (GSE) test chamber and full-field collimator. SABER was operated inside the test chamber to simulate the on-orbit operating environment. The test chamber also housed blackbody and knife-edge calibration sources. The full field collimator was attached to the exit port of the test chamber and shared vacuum and cold shield continuity. The collimator provided calibration source configurations for spatial, temporal, linearity, and relative spectral response measurements.

Infrared Spaceborne Remote Sensing XIV, edited by Marija Strojnik, Proc. of SPIE Vol. 6297, 62970V, (2006) · 0277-786X/06/\$15 - doi: 10.1117/12.692857 
For on-orbit calibration, several approaches have been used to validate and improve SABER calibration parameters. The off-axis portion of the SABER field-of-view (FOV) calibration was verified and improved through exo-atmospheric scanning of the Moon. Motion and twist effects from the TIMED spacecraft attitude were shown to be negligible. The in-flight calibration blackbody (IFCBB) and Jones Sources calibration parameters were analyzed and shown to be functioning correctly. A study of overlapping channels showed the responsivity coefficient using both the IFCBB and Jones Source calibration methods derived comparable values. Also, a Jones source study of the short wavelength channels showed two of the three Jones sources to be comparable and reproducible. Corrections for off-axis scatter and detector relaxation have been quantified and implemented. All of these analyses have contributed to accurate and dependable SABER data products.

\section{INSTRUMENT OVERVIEW}

A brief instrument overview is given to provide a summary of those areas of the instrument design that are related to calibration. The instrument design and mission objectives are described elsewhere in greater detail. ${ }^{4-7}$

SABER is a ten-channel, earthlimb-viewing sensor that measures atmospheric emissions in the $1-17 \mu \mathrm{m}$ spectral range. A functional diagram of the SABER sensor is shown in Figure 1. The optical design consists of a high off-axis rejection telescope, a single-axis scan mirror, a chopper, filters, and 10-detector focal plane elements. The focal length of the telescope is $200 \mathrm{~mm}$, with an f-number of 2 . Each detector, or channel, is spectrally filtered to a unique passband.

The atmospheric species, spectral passband, minimum radiance, maximum radiance, and equivalent blackbody temperature for each detector channel are tabulated in Table 1. The minimum radiance is expressed as noise equivalent radiance (NER). The fields of view of the ten detectors are scanned across the earthlimb by means of a one-axis scan mirror to produce vertical spectral radiance profiles of the mesosphere and lower thermosphere. The focal plane assembly is cooled to $75 \mathrm{~K}$ and the telescope to $240 \mathrm{~K}$. The earth limb is sampled over an angular range of approximately $16^{\circ}$, which allows SABER to look from cold space down to hard earth.

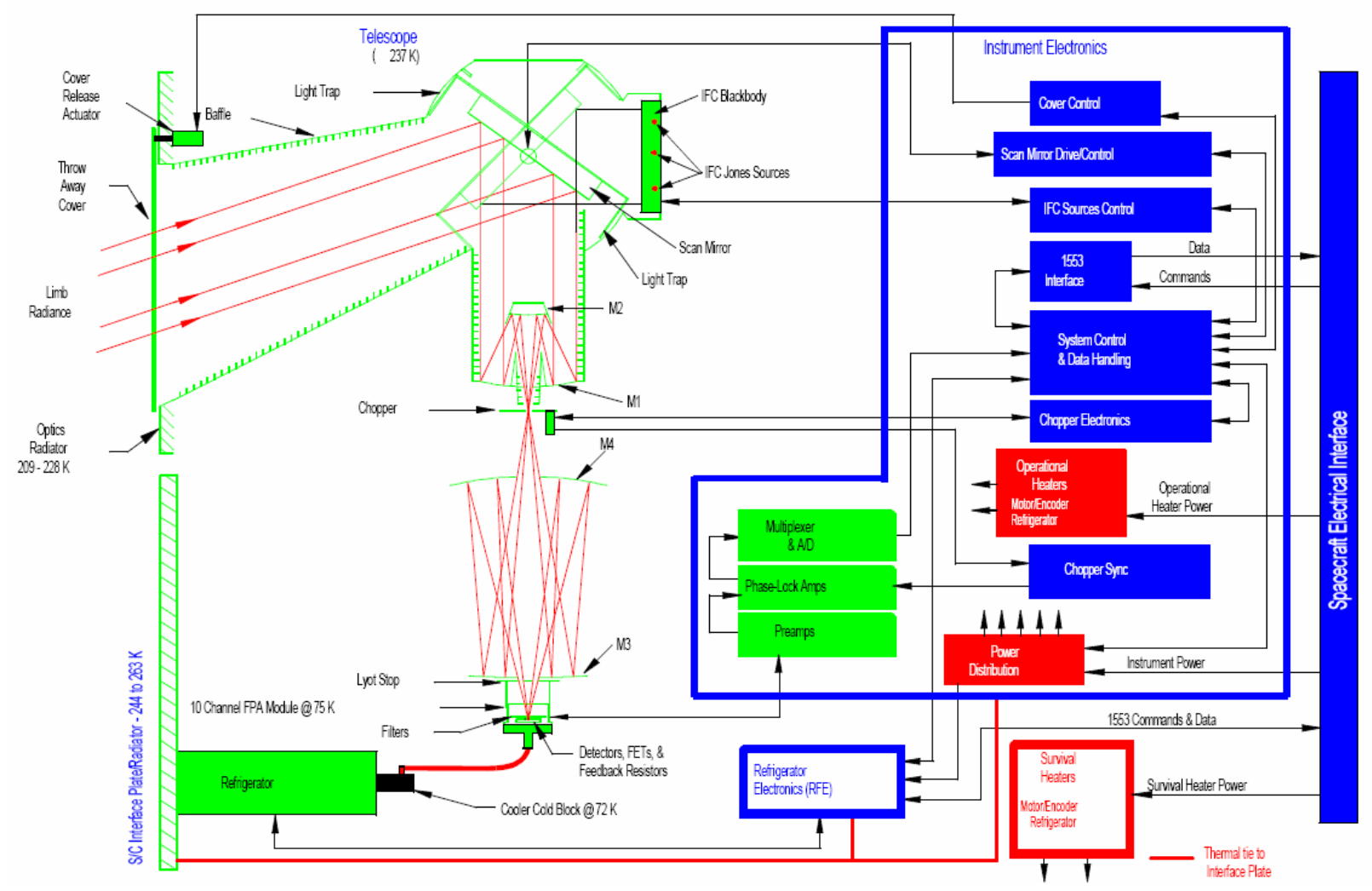

Fig. 1. SABER instrument functional diagram 
Table 1. SABER spectral passband and radiance levels

\begin{tabular}{|c|c|c|c|c|c|c|}
\hline \multirow[b]{2}{*}{$\begin{array}{l}\text { Channel } \\
\text { Number }\end{array}$} & \multirow{2}{*}{$\begin{array}{l}\text { Channel Name } \\
\text { (Based on } \\
\text { atmospheric } \\
\text { species } \\
\text { measured) }\end{array}$} & \multirow[b]{2}{*}{$\begin{array}{c}5 \% \text { Relative } \\
\text { Transmittance } \\
\text { Limits }(\mu \mathrm{m})\end{array}$} & \multicolumn{2}{|c|}{ Minimum Radiance } & \multicolumn{2}{|c|}{ Maximum Radiance } \\
\hline & & & $\begin{array}{c}\text { NER } \\
\text { Radiance } \\
\left(\mathbf{W c m}^{-2} \mathbf{s r}^{-1}\right)\end{array}$ & $\begin{array}{c}\text { Equivalent } \\
\text { Blackbody } \\
\text { Temperature } \\
\text { (K) }\end{array}$ & $\begin{array}{l}\text { Radiance } \\
\left(\mathrm{Wcm}^{-2} \mathbf{s r}^{-1}\right)\end{array}$ & $\begin{array}{c}\text { Equivalent } \\
\text { Blackbody } \\
\text { Temperature } \\
\text { (K) }\end{array}$ \\
\hline 1 & $\mathrm{CO}_{2}-\mathrm{N}$ & $14.3-15.4$ & $2.4 \mathrm{e}-8$ & 73 & $5.3 e-4$ & \multirow{7}{*}{$\begin{array}{l}\text { Hard Earth } \\
\sim 300 \mathrm{~K}\end{array}$} \\
\hline 2 & $\mathrm{CO}_{2}-\mathrm{W}$ & $13.1-17.2$ & $2.6 e-8$ & 656 & $1.9 e-3$ & \\
\hline 3 & $\mathrm{CO}_{2}-\mathrm{W}$ & $13.1-17.3$ & $2.9 e-8$ & 66 & $2.3 e-3$ & \\
\hline 4 & $\mathrm{O}_{3}$ & $8.73-9.87$ & $3.2 \mathrm{e}-9$ & 88 & $9.4 \mathrm{e}-4$ & \\
\hline 5 & $\mathrm{H}_{2} \mathrm{O}$ & $6.38-7.31$ & $2.0 e-9$ & 108 & $5.3 e-4$ & \\
\hline 6 & NO & $5.14-5.37$ & $1.4 \mathrm{e}-10$ & 126 & $4.1 e-5$ & \\
\hline 7 & $\mathrm{CO}_{2}-\mathrm{B}$ & $4.18-4.34$ & $7.7 e-11$ & 145 & $1.4 \mathrm{e}-5$ & \\
\hline 8 & $\mathrm{OH}-\mathrm{A}$ & $1.94-2.22$ & $1.3 e-10$ & 254 & $1.4 \mathrm{e}-4$ & 528 \\
\hline 9 & $\mathrm{OH}-\mathrm{B}$ & $1.56-1.74$ & $3.3 e-10$ & 324 & $2.8 e-4$ & 666 \\
\hline 10 & $\mathrm{O}_{2}$ & $1.25-1.30$ & $2.5 e-10$ & 423 & $7.4 e-5$ & 803 \\
\hline
\end{tabular}

The instrument contains IFC radiation sources to verify or update sensor calibration during on-orbit operations. Periodically during data collection, the scan mirror rotates to view cold space (400km tangent height look angle), a full aperture blackbody, and tungsten lamps. The cold space look is used to measure the sensor's dark offset, the full aperture blackbody is used to measure the responsivity of long wavelength channels (Channels 1-7), and the tungsten lamps are used to measure responsivity of short wavelength channels (Channels 8-10).

\section{OVERALL CALIBRATION APPROACH}

A subset of instrument requirements was identified that related to calibration. A comprehensive description of instrument requirements can be found in the SABER Instrument Requirements Document (IRD). ${ }^{8}$ To meet these requirements, a set of calibration measurement parameters were identified that quantified the instrument performance. Grouped into two categories, calibration equation and radiometric model, these measurement parameters were then flowed down to the calibration planning process. This process became a rather large trade study between GSE hardware and software, schedule, budget, measurement uncertainty, and risk. An uncertainty budget was established early in the calibration planning phase to track uncertainty estimates against instrument requirements throughout the planning process. If measurement parameters did not meet specifications given by the uncertainty budget, the calibration planning process was repeated until a solution met instrument calibration requirements, budget, and schedule at a minimum risk. This process was a joint effort between the science, instrument, and calibration teams.

The calibration planning effort began relatively early in the SABER program. A proposed plan was presented at the SABER systems requirement review (SRR) that provided a strawman approach for the science, instrument, and calibration teams to refine and discuss further. These teams met approximately every three months to discuss calibration issues and solutions. After a preliminary design review (PDR) and resolution of a few issues, a calibration peer review was held approximately six months prior to the SABER critical design review (CDR). At the CDR, calibration and instrument engineers from other programs were asked to participate and provide suggestions based on their experience 
with other instruments. As a result, improvements were identified and implemented. The calibration plan and GSE hardware were successfully presented at the SABER CDR without unresolved issues.

An uncertainty budget to track estimates against instrument requirements throughout the planning and development process was established early in the calibration planning phase. Efforts were directed toward reducing the largest uncertainties to give the smallest possible overall uncertainty. The calibration uncertainty budget then evolved to track uncertainties based on the most recent instrument and calibration source performance information.

On-orbit calibration is an important aspect of maintaining and updating calibration throughout mission life and, as a result of careful planning and detailed calibration, a successful minimum on-orbit calibration process was realized for SABER including (1) further updates and quantification of off-axis FOV response using lunar FOV measurements, (2) IFC sources were analyzed and shown to be functioning adequately, (3) motion and twist from the TIMED spacecraft attitude was shown to be negligible, and (4) a near real-time process of correcting data for off-axis scatter and detector relaxation for the shortwave detector channels, was implemented to improve the quality of SABER data products.

\section{CALIBRATION EQUATION}

The calibration equation is used to relate sensor output to measured radiance. The calibration equation for SABER is:

$$
L_{M, k, t}=\left(\frac{1}{\mathfrak{R}_{c h}}\right) r_{c, c h}=\left(\frac{1}{\mathfrak{R}_{c h}}\right)\left(\Upsilon_{c h, i}(s c n) G_{c h, i}\left(r_{c h, i}-O_{c h, i}\right)\right.
$$

where $L_{M, k, t}$ is measured radiance, $\mathfrak{R}_{c h}$ is peak radiance responsivity, $r_{c, c h}$ is corrected instrument response, $\Upsilon_{c h, i}()$ is off-axis extended source throughput correction, $s c n$ is scan mirror pointing angle, $G_{c h, i}$ is gain-mode normalization, $r_{c h, i}$ is detector response, $O_{c h, i}$ is sensor offset, $c h$ is channel number, and $i$ is gain mode.

The on-orbit calibration approach periodically updates the responsivity coefficient for each channel during on-orbit operations. These update are based on a space look to characterize the sensor offset and an IFC look to measure the sensor response to a known radiance. The updated responsivity coefficient can be calculated using:

$$
\Re_{c h}=\frac{r_{c, I F C}}{N_{I F C}}
$$

where $\Re_{c h}$ is the updated peak radiance responsivity, $r_{c, I F C}$ is the corrected IFC response, $N_{I F C}$ is the IFC radiance and $c h$ is the channel number.

The on-orbit scene radiance is then estimated by substituting the updated peak radiance responsivity into Equation 1 . The IFC sources were calibrated by transferring calibration of ground-based blackbodies to the IFC sources using SABER as a transfer radiometer.

\section{RADIOMETRIC MODEL}

The radiometric model relates the measured radiance from the calibration equation to the true-scene radiance presented to the sensor. This allows scientists to make better estimates of the true-scene radiance and measurement uncertainties.

As previously mentioned, the radiometric model characterizes the spatial, spectral, and temporal responsivity domains as well as random uncertainties. The spatial domain was characterized with IFOV and slit measurements, the spectral domain with system level in-band and out-of-band relative spectral response (RSR) measurements, and the temporal domain was partially verified with a radiometric step function measurement.

\section{CALIBRATION TEST FACILITY}

A calibration test facility was designed and built to measure individual parameters of the calibration equation and radiometric model. For all radiometric testing, the SABER calibration test facility, shown in Figure 2, consisted of a test 
chamber interfaced with a collimator. SABER was mounted and operated in the test chamber that also provided blackbody and knife-edge calibration sources. The full-field collimator provided source configurations for relative spectral response, IFOV, near angle scatter, short wavelength linearity, and temporal frequency response measurements. Detailed specifications for SABER GSE hardware and software are described in an SDL report entitled "SABER Ground Support Equipment Specification".,10,11

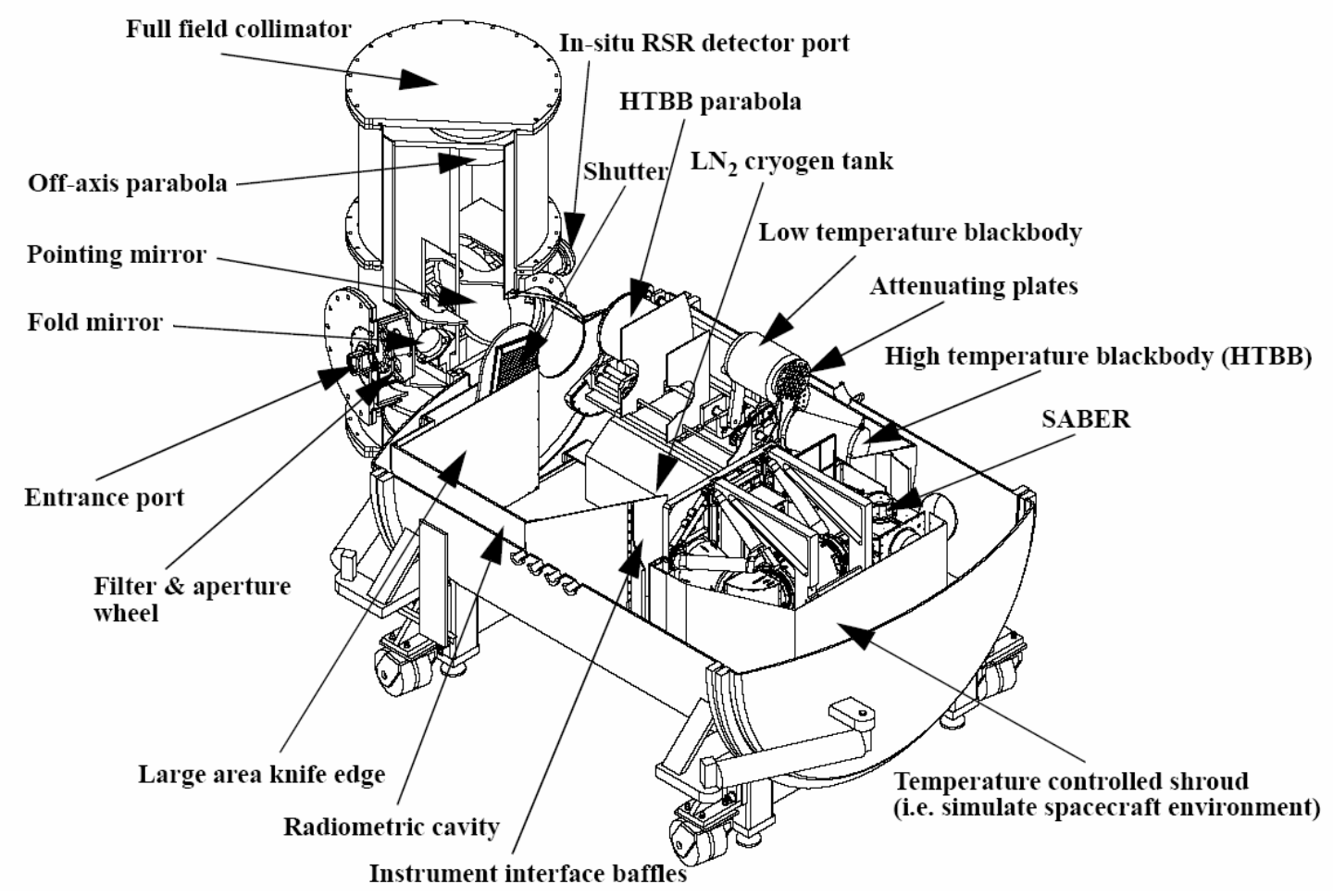

Fig. 2. Calibration test facility

\section{CALIBRATION RESULTS}

\subsection{Ground Calibration}

Detailed ground calibration results are documented in the SABER Calibration Report ${ }^{1}$ and are summarized in publication $^{2}$. These results consist of instantaneous field-of-view (IFOV), near angle scatter (NAS), temporal frequency response (TFR), relative spectral responsivity (RSR), low and high temperature blackbody, short wave linearity, and knife edge to validate large angle scatter.

\subsection{On-Orbit Calibration}

\subsubsection{Lunar FOV Off-Axis Response}

Lunar crossings through the SABER FOV at exo-atmospheric altitudes have been used to obtain accurate knowledge of the SABER off-axis response. The SABER Payload Operations Center commanded the instrument to perform several scans across the lunar surface on various occasions during the mission. Four lunar observation periods, occurring during the period January 2003 to March 2004, have been analyzed.

The lunar off-axis measurement is intended to be equivalent to scanning a bright, thick slit resulting in a low resolution FOV calibration test. Treating the Moon as a hot source against a cold background, an off-axis rejection response 
knowledge of $10^{-5}$ or better was achieved. The slowly varying off-axis portion of the in-lab high-resolution FOV measurements was corrected with low-resolution lunar off-axis response measurements. Using Channel 1 as an example, Figure 3 shows a comparison of the uncorrected in-lab FOV measurement and the corrected FOV using various lunar measurements.

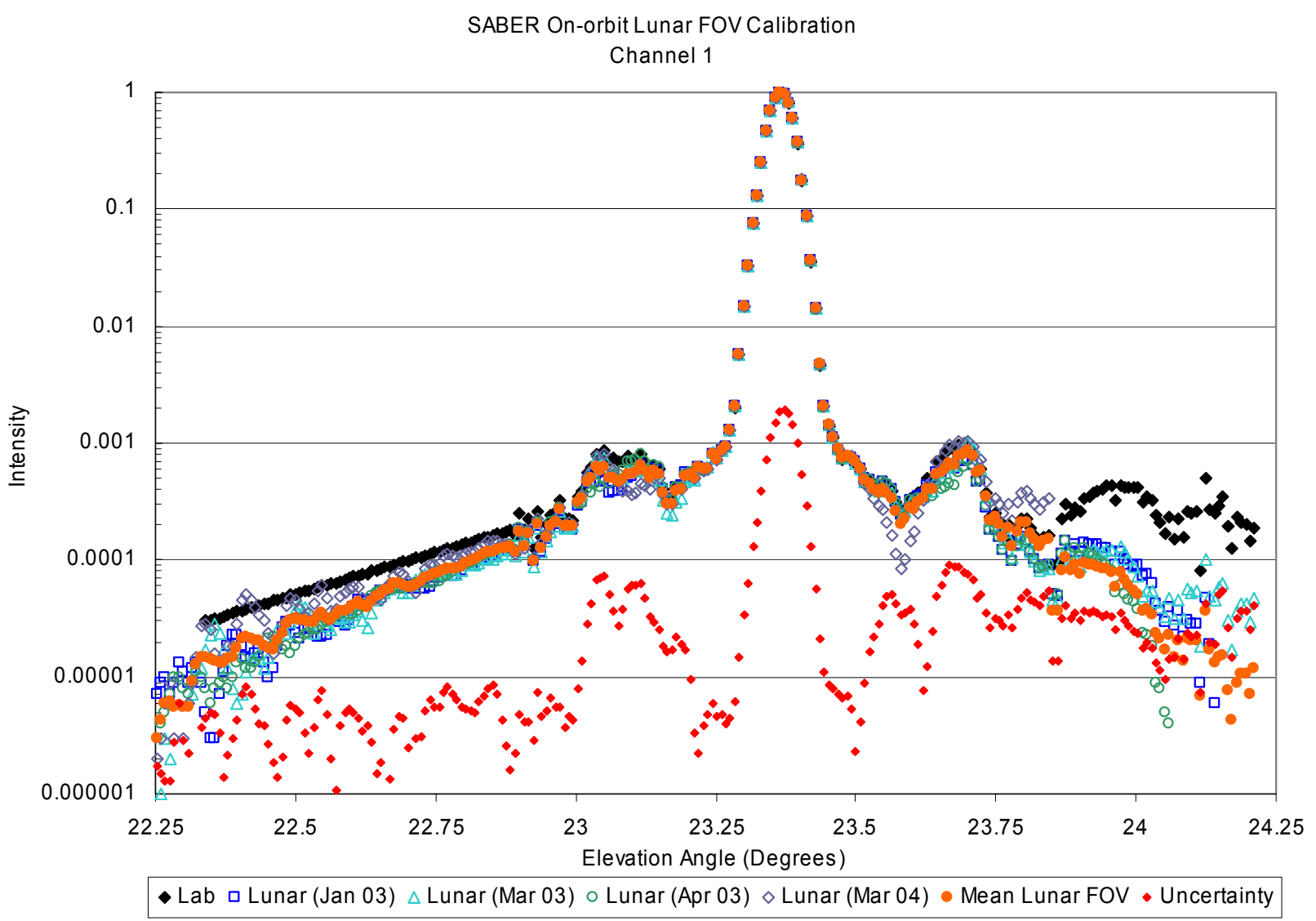

Fig. 3. Lab and lunar FOV showing $10^{-5}$ accuracy

\subsubsection{Spacecraft Motion and Twist}

Motion relative to the SABER instrument is defined as a rotation of the focal plane caused by a roll of the spacecraft about the forward x-axis. Twist is the rotation of the focal plane due to a pitch about the y-axis. Figure 4 shows the positional locations of the detector channels on the SABER focal plane and the direction of referenced roll and twist angles. Using the near-identical $\mathrm{CO} 2-\mathrm{W}$ channels 2 and 3, motion and twist were calculated using the positioning of the $\mathrm{CO} 2$ channels on the focal plane. The channels are offset by 0.81 degrees horizontally and 0.288 degrees vertically on the focal plane. With Channel 2 equivalent to Channel 3, it is possible to map the respective elevation angles of both channels to the same radiance grid. Then, with all elevation angles for an up-down pair aligned by radiance, the rate of motion could be modeled by the ratio of the difference in elevation from the down-scans to the difference in elevation from the up-scans. Unfortunately, the spectral bandpass of Channels 2 and 3 filters were not identical. For this method to work, the Channel 3 radiances have to be equivalent to the Channel 2 radiances. To solve this problem, Channel 1 (CO2$\mathrm{N})$ and Channel $3(\mathrm{CO} 2-\mathrm{W})$ were used for temperature and pressure retrieval. Since Channel 1 and Channel 3 are on the same side of the focal plane, any effect of twist and motion would be minimal. New Channel 2 radiances are simulated from this temperature and temperature retrieval. As a result, the radiance values for two identical channels at different locations on the focal plane have been achieved. 


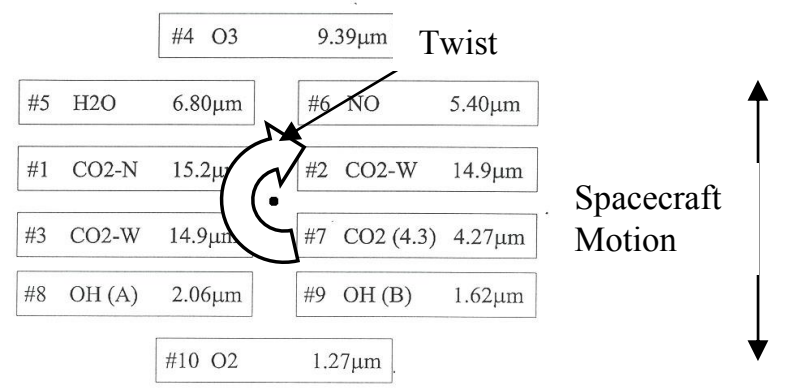

Fig. 4. SABER focal plane

Motion and twist calculations have been analyzed for several individual orbits. Table 2 shows the spacing error due to twist and mean spacecraft motion to be less than $5 \%$ of a kilometer. The timing of twist and spacecraft motion measurements were chosen to coincide with a change in attitude control from gyro-based to star tracker. The star tracker configuration was tested by TIMED from day 260 to day 275 of 2004. Since the impact from spacecraft motion and twist effects were shown to be negligible and no degradation due to star tracker was observed, it was not necessary to make corrections on SABER data products.

Table 2. Effect of twist and motion on tangent point spacing over an event

\begin{tabular}{|c|c|c|c|c|c|c|c|c|c|c|}
\hline \multicolumn{4}{|c|}{ Observation } & \multicolumn{5}{c|}{ Twist (m) } & \multicolumn{4}{c|}{ Motion (m) } \\
\hline Year & Day & Orbit & Mean & StdDev & Max & Min & Mean & StdDev & Max & Min \\
\hline 2003 & 258 & 9580 & -33.6 & 47.3 & 151.2 & -96.3 & -1.1 & 15.3 & 36.8 & -41.6 \\
\hline 2003 & 266 & 9700 & -32.3 & 42.1 & 39.4 & -88.2 & 0.5 & 19.2 & 43.1 & -49.9 \\
\hline 2003 & 276 & 9842 & -38.5 & 41.3 & 33.4 & -112.0 & -0.3 & 13.5 & 33.2 & -24.7 \\
\hline 2004 & 258 & 15000 & -47.8 & 48.5 & 61.1 & -157.2 & -2.6 & 23.3 & 67.3 & -56.1 \\
\hline 2004 & 266 & 15120 & -45.2 & 40.1 & 35.7 & -124.5 & -1.5 & 20.5 & 34.7 & -53.0 \\
\hline 2004 & 268 & 15140 & -46.5 & 45.3 & 39.3 & -151.6 & -0.8 & 25.5 & 42.8 & -101.1 \\
\hline 2004 & 276 & 15262 & -34.3 & 46.2 & 34.8 & -123.4 & -3.1 & 12.1 & 20.9 & -23.7 \\
\hline
\end{tabular}

\subsubsection{IFC Black Body and Jones Source Calibration}

SABER contains an on-board full-aperture blackbody and three small incandescent bulbs configured in a Jones source configuration (i.e. Jones sources). These sources are viewed during a calibration scan, and each source is viewed approximately three times per orbit.

For SABER processing software versions through V01.06, the IFCBB is used to calibrate Channels 1-7. Channels 6 and 7 do respond to both the Jones sources and the full aperture IFCBB. Calibration data from these channels have been used as a crosscheck between the different sources. Channels 8 and 9 are calibrated using an average of Jones Source 1 and 2 (JS1 and JS2) while Channel 10 is calibrated with JS1 and JS3. When the Jones sources are powered, they are operated with a constant current.

During ground calibration, data for Channels 6 and 7 were obtained for both the IFC full-aperture blackbody and Jones sources, and compared to ground calibration sources to obtain an absolute scale. As a crosscheck, and as part of on-orbit trending, the responsivity coefficient, $\mathrm{K}_{\mathrm{ch}}$ (counts per radiance in units of Watts $/ \mathrm{cm}^{2}$ per steradian) derived from the IFCBB calibration, was compared to the responsivity coefficient derived from each of the Jones sources. As an example, the results for Channel 7 are displayed in Table 3. These data show JS1 and JS2 to be in agreement with the IFC blackbody to $1.1 \%$ or better. This provides evidence of IFCBB and Jones source consistency and stability throughout the 
mission. JS3, however, shows a larger percent difference, as much as $20 \%$, from the IFC blackbody and may be drifting over time. Due to uncertainty of IFCJS3, at the last March 2006 meeting, the science team decided to stop using IFCJS3 in the calibration process. In future versions, Channel 10 will be switched to use JS1 and JS2, the same Jones sources as Channels 8 and 9.

Table 3. Comparison of IFCBB and Jones Source Responsivity Coefficients

\begin{tabular}{|c|c|c|c|c|c|c|c|}
\hline Channel 7 & \multicolumn{3}{|c|}{ Rch, Counts Per Radiance By Method } & \multicolumn{2}{c|}{ Percent Difference from IFCBB } \\
\hline & IFCBB & IFCJS1 & IFCJS2 & IFCJS3 & JS1 & JS2 & JS3 \\
\hline 2002 & $2.34 \mathrm{E}+10$ & $2.30 \mathrm{E}+10$ & $2.36 \mathrm{E}+10$ & $2.68 \mathrm{E}+10$ & $-1.60 \%$ & $0.94 \%$ & $14.67 \%$ \\
\hline 2003 & $2.44 \mathrm{E}+10$ & $2.43 \mathrm{E}+10$ & $2.47 \mathrm{E}+10$ & $2.84 \mathrm{E}+10$ & $-0.46 \%$ & $1.27 \%$ & $16.28 \%$ \\
\hline 2004 & $2.48 \mathrm{E}+10$ & $2.47 \mathrm{E}+10$ & $2.51 \mathrm{E}+10$ & $2.44 \mathrm{E}+10$ & $-0.24 \%$ & $1.21 \%$ & $-1.37 \%$ \\
\hline 2005 & $2.50 \mathrm{E}+10$ & $2.49 \mathrm{E}+10$ & $2.52 \mathrm{E}+10$ & $2.98 \mathrm{E}+10$ & $-0.44 \%$ & $0.95 \%$ & $19.22 \%$ \\
\hline Mean & & & & & $-0.69 \%$ & $1.09 \%$ & $12.20 \%$ \\
\hline
\end{tabular}

To further evaluate IFC performance, a time history of the Responsivity Coefficient over a three-year period was created and is shown in Figure 5. The discontinuity in the coefficients is attributed to de-icing. Responsivity is affected by sublimation forming on the detector filters. To remove the sublimation the cooler power is turned off for one day. Early in the mission this was performed every solar beta cycle. It has since been performed less frequently as ice buildup has decreased.
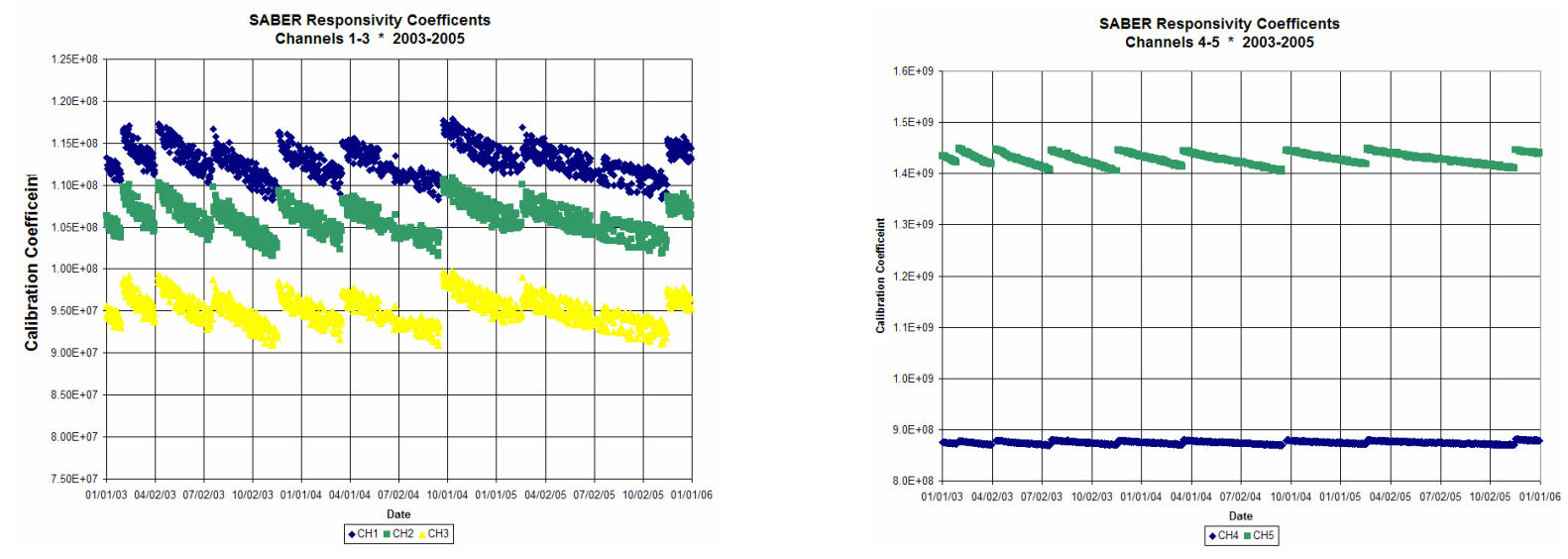

A

B 


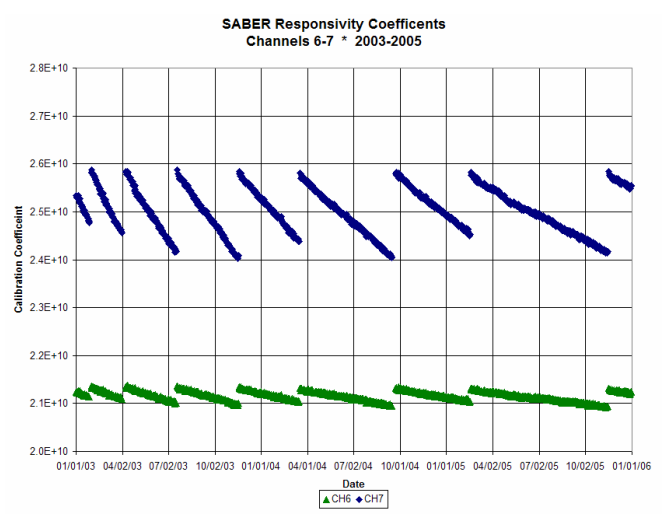

C

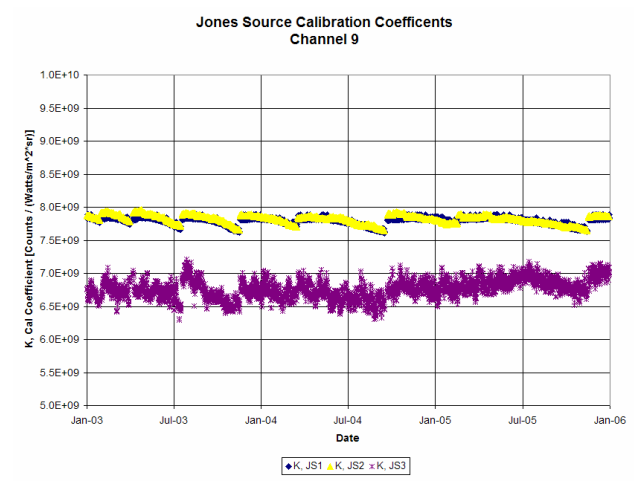

E

Fig. 5. IFCJS Calibration history (panel A, ch 1-3; panel B, ch 4-5; panel C, ch 6-7; panel D, ch 8; panel E, ch9; panel $F$, ch 10)

\subsubsection{Off-Axis Scatter}

Off-axis scatter response is noticeable in the shortwave channels. This response is due to the solar intensity at low altitudes being several orders of magnitude larger than the signals measured at higher altitudes.

To quantify this response, limb scans were separated into two categories - strong cloud and weak cloud limb-scan profiles. Profiles displaying above average scatter effects at high altitudes and high intensities at low-altitude were placed into the strong cloud profile. Those with below average scatter effects at high altitudes and those with minimal low-altitude intensities were placed in the weak cloud profile. To remove influences caused by cloud borders and daynight differences, low-altitude orbit-to-orbit limb scans were averaged. To avoid detector relaxation errors, only down scans were used in this analysis.

After the weak and strong cloud limb scan profiles have been quantified, and assuming that the entire difference of the high altitude signal is due solely to scatter, then the difference (strong-weak) profile represents the effective scatter induced by low-altitude difference radiance. The difference scan profile is log-linear at high altitudes where we expect to have no signal and can be modeled by:

$$
\mathrm{S}_{\mathrm{n}}=\int_{0}^{\mathrm{n}-1}\left(\mathrm{~A}^{*} \mathrm{R}_{(\mathrm{z})} \mathrm{e}^{-\mathrm{Bz}}\right) \mathrm{dz},
$$

where $\mathrm{B}$ is the log-linear slope at high-altitudes of the difference profile, $\mathrm{R}_{\mathrm{i}}$ is the radiance of the difference profile at $\mathrm{i}$, and $S_{n}$ is the scatter radiance at $n$. The coefficient $A$ is derived by moving it outside the integral and dividing the integral into $S_{n}$, the radiance of the difference profile at tangent point $n$. These coefficients $A$ and $B$ are then used operationally to 
calculate and correct the scatter response for every limb scan profile. Figure 6 shows limb radiance and scatter response for Channel 9, including the strong and weak cloud limb radiance profiles, the difference limb profile, the scatter response correction (effect) for weak and strong cloud limb profile, and the corrected (derived) profile for both statistical sets.

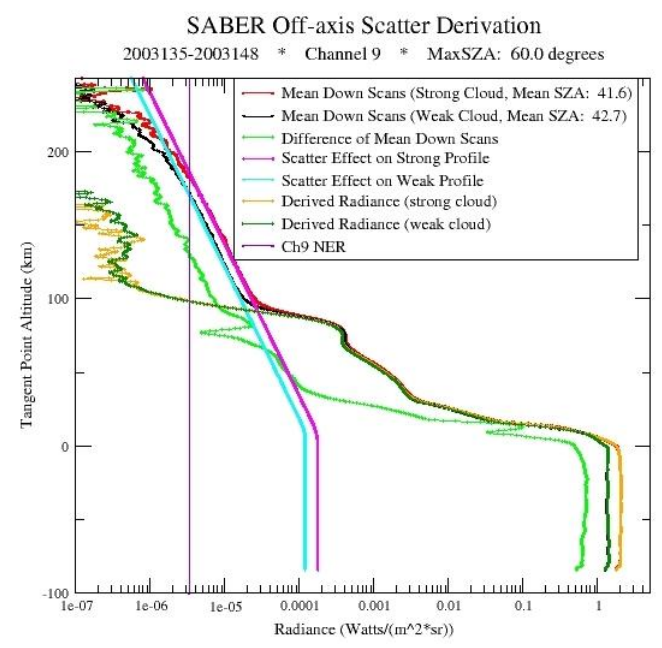

Fig. 6. Off-axis scatter correction

Operationally, a limb scan radiance profile is created by averaging all events within three minutes to either side of each limb scan measurement. The scatter correction is calculated using the derived coefficients along with the field-of-regard (FOR) profile and subtracted off of the subject profile. The results bring the short wavelength channels to the noise much faster and at what we believe to be a more realistic rate.

\subsubsection{Detector Relaxation}

Detector relaxation response (i.e. residual detector response after the magnitude of the true scene flux has decreased) has been observed in the shortwave channels. For down scans (scanning from space to earth), the radiance increases in magnitude, making detector relaxation negligible. However, for up scans (from hard earth to space) the radiance is decreasing and a correction for detector relaxation is needed to reduce uncertainty.

The method to quantify this effect is similar ${ }^{12}$ to the off-axis scatter as discussed in the previous section. Instead of using the strong and weak cloud limb profiles as in the off-axis scatter analysis, statistical data sets are separated into up and down scans. The difference (up-down) profile is log-linear and was modeled by:

$$
\mathrm{H}_{\mathrm{n}}=\int_{0}^{\mathrm{n}-1}\left(\mathrm{~A}^{*} \mathrm{R}_{(\mathrm{z})} \mathrm{e}^{-\mathrm{B} z}\right) \mathrm{dz},
$$

where $B$ is the log-linear slope at high-altitudes of the difference profile, $R$ is the radiance of the up scan profile, and $\mathrm{H}_{n}$ is the radiance attributed to detector relaxation. The coefficients for the detector relaxation correction are derived in exactly the same manner as for off-axis scatter except that the up scan radiance profile is used instead of the difference profile. This is due to the assumption that the down scan profile is the true profile, whereas the weak cloud profile still contained scatter error.

Operationally, the correction is derived using individual up and down scan pair profiles to quantify coefficients of Equation 4. Figure 7a shows radiance earth limb scan profiles for Channel 9 for up scans with and without detector relaxation correction. The radiance difference between up and down scans with and without detector relaxation correction is shown in Figure $7 \mathrm{~b}$. These data show that detector relaxation causes a significant difference between up and down scans and the correction makes the two measurements significantly more consistent. 


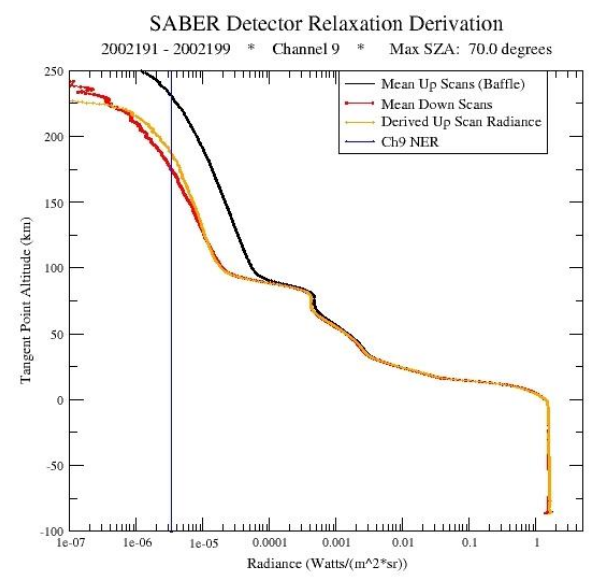

A

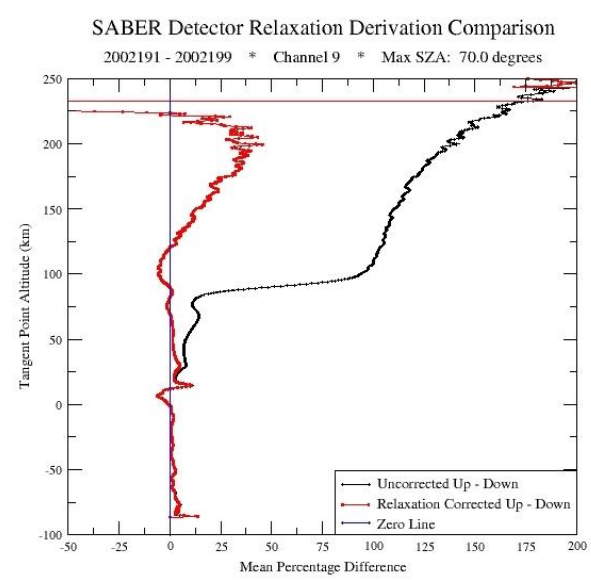

B

Fig. 7. Detector relaxation correction (panel A) and difference (panel B)

\section{SUMMARY}

SABER is a 10-channel infrared $(1.27-17.0 \mu \mathrm{m}$ wavelength) radiometer designed to measure atmospheric infrared emissions from the earthlimb. Ground calibration for the SABER instrument was complete in September 1999. Calibration measurement parameters quantify the instrument performance and are grouped into two categories, calibration equation and radiometric model. The calibration equation relates sensor output to measured radiance. The radiometric model describes the spatial, spectral, and temporal domains of the instrument.

Calibration planning began while the SABER instrument was in design. Technical interchange meetings, conducted approximately every three months, provided a platform for science, instrument, and calibration teams to discuss calibration issues and solutions. An engineering calibration was conducted prior to final calibration testing to verify test configurations, verify operation of GSE and data collection software, finalize data collection procedures, provide preliminary data to evaluate instrument performance, and verify test procedures.

A calibration test facility was designed and built to measure individual parameters of the calibration equation and radiometric model. For all radiometric testing, the SABER calibration test facility consisted of a test chamber interfaced with a collimator. SABER was mounted and operated in the test chamber, which also provided blackbody and knife edge calibration sources. The full-field collimator provided source configurations for relative spectral response, IFOV, near angle scatter, short wavelength linearity, and temporal frequency response measurements.

An uncertainty budget was established early in the calibration planning phase to track uncertainty estimates against instrument requirements throughout the planning and development process. Ground calibration efforts were directed at reducing the largest uncertainties to give the smallest possible overall uncertainty. The calibration uncertainty budget then evolved to track uncertainties based on the most recent instrument and calibration source performance information.

On-orbit calibration is an important aspect of maintaining and updating calibration throughout mission life and, as a result of careful planning and detailed calibration, a successful on-orbit calibration process was realized for SABER including (1) further updates and quantification of off-axis FOV response using lunar FOV measurements, (2) IFC sources were analyzed and shown to be functioning adequately, (3) motion and twist from the TIMED spacecraft attitude was negligible and (4) a near real time process of correcting data for off-axis scatter and detector relaxation for the shortwave detector channels was implemented to improve the quality of SABER data products.

\section{ACKNOWLEDGEMENTS}

Work reported in this paper has been supported by NASA contracts NAS1-20080, NAS1-20476, and NAS1-97155. 


\section{REFERENCES}

1. SDL/99-155, SABER Ground Calibration Report. Space Dynamics Laboratory. 2000.

2. J. Tansock, S. Hansen, K. Paskett, A. Shumway, J. Peterson, J. Stauder, L. Gordley, M. Wang, J. Melbert, J. Russel, M. Mlynczak, "SABER Ground Calibration," International Journal of Remote Sensing, Vol. 24, NO. 2, 403-420. 2003.

3. C. Wyatt. Radiometric calibration: theory and methods. New York: Academic Press, New York. 1978.

4. R. Esplin, C. Batty, M. Jensen, D. McLain, J. Stauder, S. Jensen, C. Stump, D. Robinson, and J. Dodgen, "SABER instrument overview." Proceedings, International Society of Optical Engineering, 2268, 207-219. 1994.

5. R. Esplin, L. Zollinger, C. Batty, S. Folkman, M. Roosta, J. Tansock, M. Jensen, J. Stauder, J. Miller, M. Vanek, and D. Robinson, "SABER instrument design update." Proceedings, International Society of Optical Engineering, 2553, 253-263. 1995.

6. J.M. Russell III, M.G. Mlynczak, L.L. Gordley, J. Tansock, and R. Esplin, 1999, An overview of the SABER experiment and preliminary calibration results. Proceedings, International Society of Optical Engineering, 3756, 277-288. 1999.

7. SDL/97-076, SABER Critical Design Review. Space Dynamics Laboratory. 1997.

8. SDL/95-006, SABER instrument requirements document. Space Dynamics Laboratory. 1995.

9. SDL/97-070, SABER ground support equipment documentation. Space Dynamics Laboratory). 1999

10. SDL/98-112, SABER GSE low-temperature blackbody calibration source. Space Dynamics Laboratory. 1998,

11. Instruction manual for model M360XHTVBB blackbody calibration source. Mikron Instrument Company. 1999.

12. M. Ern, D. Offermann, P. Preusse, K. Grossmann, and J. Oberheide, "Calibration procedures and correction of detector signal relaxations for the CRISTA infrared satellite instrument," Applied Optics, Vol. 42, No. 9. 2003. 